\title{
BMJ Open Can extranodal tumour deposits be diagnosed on MRI? Protocol for a multicentre clinical trial (the COMET trial)
}

\author{
Amy C Lord (D) , ${ }^{1,2}$ Brendan Moran, ${ }^{3}$ Muti Abulafi, ${ }^{2}$ Shahnawaz Rasheed, ${ }^{1}$ \\ Iris D Nagtegaal, ${ }^{4}$ Monica Terlizzo, ${ }^{1}$ Gina Brown ${ }^{5}$
}

To cite: Lord AC, Moran B, Abulafi M, et al. Can extranodal tumour deposits be diagnosed on MRI? Protocol for a multicentre clinical trial (the COMET trial). BMJ Open 2020;10:e033395. doi:10.1136/ bmjopen-2019-033395

- Prepublication history and additional materials for this paper is available online. To view these files, please visit the journal online (http://dx.doi org/10.1136/bmjopen-2019bmjopen-2019-033395).

Received 06 August 2019 Revised 15 July 2020

Accepted 21 August 2020

Check for updates

(c) Author(s) (or their employer(s)) 2020. Re-use permitted under CC BY-NC. No commercial re-use. See rights and permissions. Published by BMJ.

${ }^{1}$ Department of General Surgery, Royal Marsden NHS Foundation Trust, London, UK

${ }^{2}$ Department of General Surgery, Croydon University Hospital,

Croydon, UK

${ }^{3}$ Department of General

Surgery, Basingstoke and North Hampshire NHS Foundation

Trust, Basingstoke, UK

${ }^{4}$ Department of Pathology, Radboud University Medical

Centre, Nijmegen, The

Netherlands

${ }^{5}$ Department of Radiology, Royal Marsden NHS Foundation Trust, London, UK

Correspondence to

Dr Amy C Lord;

amylord@nhs.net

\section{ABSTRACT}

Introduction Tumour deposits (TDs) are a poor prognostic marker when seen on pathology, and are worse than lymph node metastases (LNMs). They are now being reported on MRI as discontinuous nodules of extramural venous invasion but this diagnosis has not been validated and it is unclear how it correlates with the diagnosis of TDs on pathology.

Methods and analysis This is a prospective interventional clinical trial which aims to directly map the location of TDs on MRI and correlate what is seen on MRI with the pathology findings at each location. All patients with rectal cancer undergoing resectional surgery are eligible (including those undergoing preoperative therapy). The primary outcome is the prevalence of TDs seen on pathology. Secondary outcomes are to assess radiological and pathological interobserver agreement, assess the effect of TDs on prognosis and carry out exploratory work looking at differences between TDs and LNMs. The estimated sample size is 100 to detect a twofold increase in the pathological diagnosis of TD when MRI mapping is used.

Ethics and dissemination Ethical approval has been granted from the South Central-Hampshire B Research and Ethics Committee (IRAS 217627). The study will be carried out under standard operative procedures within the Royal Marsden Hospital.

Trial registration number NCT03303547.

\section{INTRODUCTION}

\section{Background}

Predicting prognosis in rectal cancer and determining indications for neoadjuvant treatment are ongoing challenges for colorectal multidisciplinary teams (MDTs). Staging is almost universally based on the tumour node metastasis (TNM) system which, despite multiple changes and increasing levels of subclassification and complexity, is still flawed in its ability to stratify patients and predict prognosis. ${ }^{1-3}$ Determining which patients will benefit most from neoadjuvant and adjuvant therapy is still controversial; UK guidelines from the National Institute
Strengths and limitations of this study

- This is the first attempt to provide direct correlation between MRI and pathology in diagnosing tumour deposit (TD).

- Stringent pathology reporting criteria will be applied to ensure robust definitions of TDs and interobserver variation will be assessed.

- Nodule by nodule comparison between MRI and pathology will be possible due to the mapping process used.

- The sample size may not be sufficient to detect the effects of TD on survival as the study is powered to detect increased pathology detection rate.

of Clinical Excellence are open to interpretation $^{4}$ and there is significant geographical variation in the way patients are managed.

Extranodal tumour deposits (TD) were first described by Gabriel et a $\tilde{l}$ in 1935 but despite the 80 intervening years, there is still ongoing controversy about their prevalence, nature, effect on prognosis and how they should be differentiated from lymph nodes (LNs). The definition of TD within the TNM system is one of the major changes to have taken place over the years; the last four TNM editions have differed significantly in their classification. TNM 5 used size criteria where TD of less than $3 \mathrm{~mm}$ were counted in the $\mathrm{T}$ stage and those over $3 \mathrm{~mm}$ were counted as LN. ${ }^{6} \mathrm{TNM}$ 6 changed to morphological criteria where if TD were rounded they were counted as LN and otherwise as part of the T stage. ${ }^{7}$ TNM 7 is the first edition to call for evidence of $\mathrm{LN}$ architecture on pathology in order to classify a nodule as an LN. If there is no LN architecture present, the nodule should be classified within a new subcategory: 'N1c'. This was defined as 'separate nodules or deposits of malignant cells in the perirectal or pericolic fat without evidence of residual lymph node 
tissue. 8 TNM 8 has not changed this definition but has added an additional stipulation that lesions with evidence of lymphatic, vascular or perineural invasion should be excluded from the category. This is not evidence based or justified. Experts have argued that there is not sufficient evidence to include TD as part of the $\mathrm{N}$ stage at all and that there is a lack of reproducibility. ${ }^{9}$ For this reason, many pathologists in the UK do not use TNM 7 and are still using TNM 5 . This means that TD are either included in the $\mathrm{T}$ or the $\mathrm{N}$ stage but there is no requirement to report them separately.

There is growing evidence that TD have a significant adverse effect on both overall and disease free survival. A meta-analysis by Nagtegaal ${ }^{10}$ noted that TD were independently associated with a poor prognosis. A more recent meta-analysis of 26 studies carried out at the Royal Marsden found when TD were present there was a pooled HR for adverse overall survival of 1.63 (95\% CI 1.44 to 1.61 ) and 1.77 (95\% CI 1.37 to 2.11 ) for disease free survival. Our meta-analysis also found a strong link between TD and extramural venous invasion (EMVI) which would be consistent with Gabriel's original description but has not been previously reported. We believe that TD and EMVI are a continuation of the same process where TD represent a more advanced form of EMVI with nodules closely related to vessels but not in continuity with the tumour itself. These nodules could be seen as metastases in transit which would make their association with poorer survival and higher rates of recurrence not surprising.

MRI can accurately identify pathological markers of poor prognosis preoperatively to allow risk stratification and aid decision-making. The Colorectal Cancer Imaging Research Team at the Royal Marsden Hospital has a track record of identifying pathological markers on MRI and proving their prognostic value. Initially, the Magnetic Resonance Imaging in Rectal Cancer European Equivalence Study (MERCURY) trial proved the ability of MRI to predict involvement of the circumferential resection margin $(\mathrm{CRM})$ and therefore prognosis ${ }^{11}$; more recently the prognostic accuracy of EMVI diagnosis on MRI has also been validated. ${ }^{12-14}$ Indeed, MRI may in some cases be a better modality to visualise pathological changes than pathology itself; EMVI being an example of this. The ability to visualise the vein in multiple dimensions gives MRI a distinct advantage over pathology. Pathology techniques are inherently subject to sampling limitations which may result in underdiagnosis and the detection rate has been shown to be significantly lower. ${ }^{15}$ We believe that, similarly, TD can also be identified on MRI more easily than on pathology and hypothesise that we will be able to prove the prognostic accuracy of identifying this feature on imaging in the same way as we have with EMVI.

Currently TD are already being diagnosed on MRI at the Royal Marsden Hospital but this diagnosis has not been prospectively validated against that of pathology, which is the gold standard, and has not previously been described in the literature. TD are defined as the appearance on
MRI of nodules arising within venous channels, identified as signal void vessels, in continuity with major venous branches within the mesorectum and discontinuous with the main tumour. This novel prognostic marker is included in the MRI report as mrTD. This explicit relationship between EMVI and TD has not been described on pathology but the nature of assessing a single discrete slice through a deposit means this would be very difficult to see. The three-dimensional nature of MRI means this relationship can be seen very clearly. Preliminary work within the South West London Cancer Network has shown MRI diagnoses TD in 51\% of patients with rectal cancer $(37 \%$ of which is still visible after selected patients have undergone neoadjuvant therapy) compared with 13\% reported on pathology. The COncordance in MRI and Pathology Diagnosis of Extranodal Tumour Deposits (COMET) study hypothesises that this discrepancy is first due to the use of TNM 5 to classify TD, meaning that they are reported as LN or as an extension of the T stage but not recorded separately. Second, the limited ability of pathology to visualise the entire mesorectum in three dimensions (as is possible using imaging) means that TD are more likely to be missed.

In the literature, the prevalence of TD varies significantly. In the 26 studies included in our meta-analysis, the prevalence ranged from $10.2 \%$ to $44.2 \%$ (median $21.3 \%) .{ }^{16}$ This is likely to be in part due to patient selection but also problems with sampling techniques and classification. Of note, there was a much higher prevalence in those studies that used additional step sectioning rather than standard techniques. There is known to be a degree of interobserver variability when differentiating TD from $\mathrm{LN}^{17}$ and none of the TNM editions provide objective criteria which must be present to call a nodule an LN, only specifying that 'lymph node architecture' should be present and not defining exactly what this constitutes. Some of the features commonly used by pathologists to define an LN, such as round shape, may equally be features of an TD. Other features such a LN capsule may be difficult to distinguish from a vessel wall without the use of staining. We believe the criteria for defining a LN need to be far more objective and specific than is currently the case.

Molecular pathology techniques are widely used in current practice and can give further information about tumour behaviour (eg, KRAS testing to determine likely response to certain chemotherapy agents). Knijn et $a l^{18}$ have shown very low discordance in KRAS mutation between the primary tumour and distant disease $(<5 \%$ for liver metastases, $7 \%$ for lung metastases) but high discordance between LNs and the primary tumour of 20\%-40\%. The concordance of KRAS mutations and other markers in EMVI and TD has never been reported. The COMET study hypothesises that the profile of EMVI and TD will have a higher concordance with the primary tumour and any metastases that develop than that of LNs because this is the primary route of metastasis. The study also hypothesises that the concordance between EMVI 
and TD will be high. If this hypothesis is correct, it would allow a better understanding of the metastatic process and allow better prediction of those likely to suffer local and distant failure. This would in turn take us a step closer to cancer treatment that can be personalised to each patient taking multiple factors into consideration to determine individual risk.

Given the prognostic importance of TD shown in our meta-analysis, it is imperative that these lesions are accurately reported on pathology so that their presence can be taken into account when making treatment decisions, and furthermore in future clinical trials when stratifying which patients are most likely to benefit from neoadjuvant and adjuvant therapy. In order to accurately assess and quantify the individual effects that factors such as LN status, TD and EMVI have on prognosis, the first step is to ensure consistent reporting of their presence. Identifying adverse prognostic features before surgery rather than on pathology has distinct advantages in that it contributes to planning of surgery and also decisions about neoadjuvant therapy. If we are able to validate the MRI diagnosis of TD, this has important implications both for future research and clinical practice.

\section{Rationale}

Proving that the lesions seen on MRI are TD will alter the way we stage patients currently and due to the known association of TD with a poor prognosis, could help guide decisions about the use of neoadjuvant therapy. Additionally, if patients are being underdiagnosed on pathology they may be missing out on adjuvant therapy which could have reduced their risk of recurrence. The TNM system will most likely need to be modified if we can prove the prognostic importance of this MRI finding.

\section{Primary objectives}

- To determine whether the prevalence of TD on pathology is found to be higher if MRI mapping is used.

\section{Secondary objectives}

- To determine whether lesions classified as TD on MRI correspond to the pathological diagnosis of TD.

- To determine the effect of MRI and pathological diagnosis of TD on disease free survival (at 1, 3 and 5 years), overall survival (at 1, 3 and 5 years) and time to local recurrence.

- To determine the concordance in molecular pathology between primary tumour and TD, LN and metastases.

- To objectively record the features seen which help distinguish an LN from a TD and attempt to refine and clarify the definitions used in pathology.

- To assess interobserver agreement between the local pathologist and the central reviewing pathologist.

- To assess interobserver agreement between the local radiologist and central reviewing radiologist.

- To assess the correlation between the finding of a nodular invasive border of the primary tumour on
MRI and pathology findings of tumour budding reported.

\section{Hypotheses}

- The prevalence of TD is actually far higher than the $13 \%$ reported by routine pathology, and may be closer to the $37 \%$ seen on imaging.

- As well as following standard pathological procedures, additional sections will be taken from the area where mrTD are thought to be present on MRI. This will upstage some patients and potentially lead to a change in treatment strategy.

- There will be greater odds of having 'equivocal' nodules with a paucity of features to suggest LN origin in the mrTD positive group.

- TD are closely related to EMVI and are a continuation of this process.

- There will be higher molecular pathology and genetic concordance between primary tumour and TD than primary tumour and LN.

- MRI diagnosis of TD will accurately predict poorer prognosis.

- TD, together with EMVI, are more important than LN status in predicting recurrence and survival.

- TD could potentially be an important indication for neoadjuvant and adjuvant therapy.

- Finding a large number of LNs together with an absence of TD may be associated with improved prognosis as it signals an appropriate immune response.

\section{METHODS}

\section{Study design}

This is a prospective interventional, UK multicentre study.

\section{Radiology}

Scans will be standardised and reporting criteria will be predefined. Quality assurance of the scans will be monitored by the investigators.

Local radiologists will be asked to complete a study imaging case report form (CRF) for the baseline MRI scan, and postpreoperative treatment MRI scan, if applicable, which will include standard staging information and, additionally, information on the presence of TD (mrTD). Before site initiation, local study radiologists will be given training in identifying mrTDs and asked to complete an assessment for quality assurance purposes. Only radiologists achieving a kappa score of 0.7 will be allowed to participate in the study. Radiologists failing the assessment will be given additional training until the standard can be met.

Prior to surgery, imaging CRFs and preoperative MRI scans will be sent to the Royal Marsden so that the COMET chief investigator (or one of her radiology registrars) can create an array of images and complete a central review CRF (see figures 1 and 2) from the preop MRI scan. Each mrTD will be labelled (eg, E1, E2, E3). These images will be sent to the site study pathologist prior to histopathology processing of the specimen. 


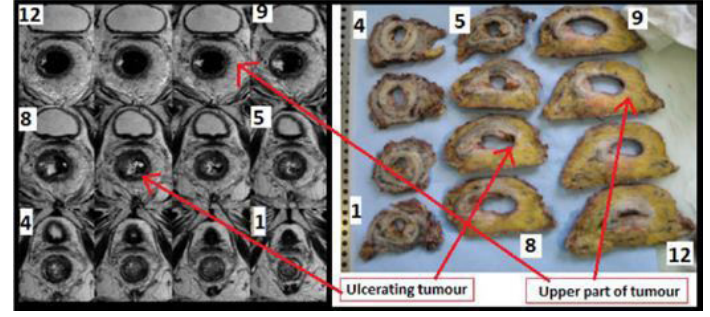

Figure $1 \mathrm{MRI}$ array and photographs of pathology specimen illustrating how mapping will be carried out.

\section{Histopathology}

Histopathology assessment will be carried out according to the Royal College of Pathologists guidelines for reporting of colorectal cancers.

As well as following standard pathological procedures, additional sections will be taken from the area where mrTD are thought to be present on MRI, and these will be kept separately from LNs and clearly labelled. Deeper sections and elastin staining will only be carried out if the pathologist would do this as standard practice (ie, it will not be requested as part of the trial). The pathologist will assess the specimen and report whether TD are present using a proforma. Tumour differentiation, CRM, $\mathrm{T}$ and $\mathrm{N}$ stages and the presence of EMVI will also be reported as standard. All pathology reporting will adhere to the Royal College of Pathologists Dataset Requirements for Reporting in Colorectal Cancer ${ }^{19}$ to ensure

\section{COMET Tumour Deposit Central Review}

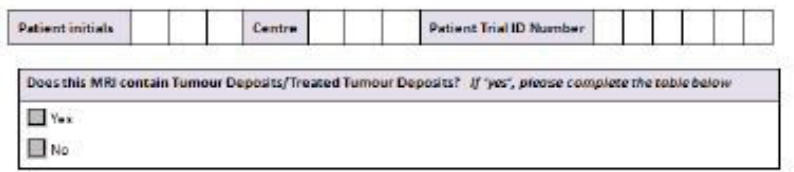

\begin{tabular}{|l|l|l|l|}
\hline Series & Number & Slice Number & Clock Position \\
\hline & & & \\
\hline & & & \\
\hline & & & \\
\hline & & & \\
\hline & & & \\
\hline & & & \\
\hline & & & \\
\hline & & & \\
\hline & & & \\
\hline & & & \\
\hline & & & \\
\hline
\end{tabular}

Pschologot signsture

orte:

Figure 2 Central pathology reporting case report form illustrating how MRI and pathology findings will be matched up. COMET, COncordance in MRI and Pathology Diagnosis of Extranodal Tumour Deposits. standardisation between sites. A photograph of the tissue slices on a numbered grid will be sent into the trial office and used for mapping purposes. This should be accompanied by a completed pathology CRF and anonymised histopathology report.

All tissue (including slides, blocks and cassettes) will be sent to the Royal Marsden COMET trial team for central review and to allow for further assessment with additional examination, staining and DNA extraction for genetic testing and KRAS, NRAS, BRAF and PIK3CA analysis. Due to the known problems with interobserver variability in distinguishing TD from $\mathrm{LN}(17)$, we will attempt to objectify this by specifically asking the site pathologist and central pathologist to identify whether any features of a LN are present for each nodule examined as well as recording the presence of vascular and neural invasion. Features which would be specific to the lesion being an $\mathrm{LN}$ will be given an ' $\mathrm{L}$ ' score and those which suggest a lesion of extranodal origin will be given an ' $E$ ' score. If the patient subsequently presents with metastatic disease and a pathology specimen is available (either in the form of a resection or a biopsy), this will also be obtained for the same testing. Scanned-in images of slides taken from the nodules will be used for their LN assessment and central review.

After analysis, all tissue samples will be returned to the Royal Marsden and kept in the Biobank for use in future research. All movement of tissue samples between centres will be logged on a secure database so an accurate account of where the samples are is available throughout the course of the study.

\section{Follow-up}

Patients will undergo standard clinical follow-up for a minimum of 5 years from the date of surgery. Clinic visits, imaging, blood tests and endoscopic follow-up will be carried out according to local protocols (see study flow chart; figure 3).

\section{Endpoints}

For all endpoints (with the exception of secondary endpoint number 4, inter observer agreement between local and central pathologists), the results from the central review for both pathology and MRI will be used.

\section{Primary end point}

The primary endpoint will be the prevalence of TD on MRI confirmed by reported TD and equivocal nodules without evidence of $\mathrm{LN}$ architecture on pathology.

\section{Secondary endpoints}

1. Concordance between MRI and pathology will be determined.

2. The $\mathrm{L}$ and $\mathrm{E}$ scores for the nodules indicated on MRI will be reported.

3. The presence of $\mathrm{L}$ and $\mathrm{E}$ features which were only visible on deeper sections or using elastin staining will be reported. 


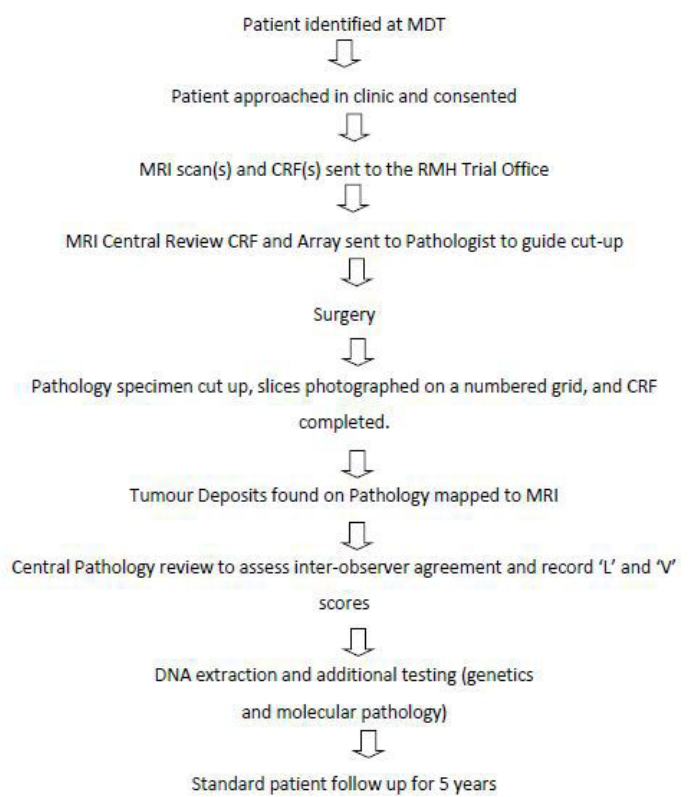

Figure 3 Study flow chart. CRF, case report form; MDT, multidisciplinary team; RMH, Royal Marsen Hospital.

4. Interobserver agreement between the local pathologist and the central reviewing pathologist (who will be using additional techniques) will be determined.

5. 1, 3 and 5-year overall survival (OS) and diseasefree survival (DFS) and time to local recurrence will be reported in four groups of patients: TD+/ $\mathrm{LN}+, \mathrm{TD}+/ \mathrm{LN}-, \mathrm{TD}-/ \mathrm{LN}+$ and $\mathrm{TD}-/ \mathrm{LN}-$. This will be done separately for both MRI and pathology diagnosis.

6. EMVI prevalence and association with TD, LN status, LN yield, T stage and CRM status will also be reported. This will allow analysis of whether TD have independent prognostic significance when other known prognostic factors are controlled for.

7. Interobserver agreement between the local radiologist and the central reviewing radiologist will be determined.

8. The presence of TD on the pretreatment MRI will also be reported to assess the prognostic significance of this.

9. Concordance in molecular pathology between primary tumour and TD, LN and metastases will be reported.

10. The correlation between the finding of a nodular invasive border on MRI and pathology findings of tumour budding will be reported.

\section{Inclusion/exclusion criteria}

Inclusion criteria

- Primary adenocarcinoma of the rectum (proven by biopsy).

- Amenable to surgical resection.

- Disease spread assessed on CT and MRI scans.

- Patients having primary surgery and those undergoing neoadjuvant treatment will be included.
- All must have had a baseline staging MRI and those undergoing neoadjuvant therapy must also have had a post-treatment MRI.

- Patients aged 18 years and over.

Exclusion criteria

- Patients with recurrent tumours.

- Synchronous tumours.

- Unable to have an MRI scan (eg, pacemaker, contrast allergy, severe claustrophobia).

- Under the age of 18 years.

- Unable to give informed consent.

\section{Withdrawal criteria}

Patients will be withdrawn from the trial for the following reasons:

- Surgery is not carried out (eg, due to illness or death between the decision to operate and the day of surgery).

\section{Intervention}

The proposed intervention will be additional radiological and pathological assessment and the reporting of supplementary diagnostic information which would not otherwise have been available. This may affect treatment according to local MDT protocols and also affect the provision of prognostic information to patients in subsequent discussions.

\section{Data acquisition}

Patient details including demographics will be recorded electronically on a secure clinical trial database. Data will be anonymised using a unique patient identification number (study ID). CRFs will be used to record data from the MRI scans and pathology reports. Pathology and radiology results and treatment data will also be entered into the database which will remain on the RMH network. Patients will only be identifiable by patient number and date of birth. All laboratory work will be kept on a secure database, identifiable by patient number and date of birth only.

Study organisation/trial monitoring and management strategy Professor GB will have overall delegated responsibility for the study as the chief investigator under the Research Governance Framework Guidelines, Good Clinical Practice.

The Colorectal Cancer Imaging Research Team at the Royal Marsden Hospital will be responsible for all delegated sponsor trial management duties as well as the running of COMET at the Royal Marsden site. Duties will include: registering patients, the day-to-day running of the study, data collection, CRF design, management of amendments and site set-up and monitoring the study.

The statistician will have responsibility for data management and integrity. Professor GB will be the principal investigator at the Royal Marsden Hospital site.

There are no interventions requiring risk assessment. The only intervention taking place is the recording of 
additional diagnostic information during radiological and pathological assessments. Standard MRI safety procedures will be conducted as per trust guidelines.

\section{Evaluation of outcome}

The outcomes will include the prevalence of TD on MRI and TD and equivocal nodules on pathology. Equivocal nodules will be further investigated to objectively define features suggestive of LN or non-LN origin. Concordance in molecular pathology and genetic heterogeneity will also be reported. Survival outcomes will be assessed after the follow-up periods defined above.

\section{Adverse events}

Patients on the study may experience adverse events which may or may not be attributable to the study procedure. However, the study will not be collecting, recording or reporting any adverse events reported on the study because the intervention in this trial is simply an improved method of staging and will not mandate or advocate any specific changes to a patient's clinical management.

\section{Statistical considerations}

\section{Sample size}

There is no existing research to base a sample size calculation on. We used our own retrospective data (described above) and predicted that we would be able to at least double the prevalence of TD and equivocal nodules on pathology from $13 \%$ to $26 \%$ in this trial. The justification for also including equivocal nodules is that whether these are $\mathrm{LN}$ or TD is irrelevant based on current treatment models as both would be eligible for adjuvant treatment and are therefore an important finding which would upstage the patient.

The study uses an Ahern single stage phase II design with a one-sided $\alpha$ of 0.05 . The null hypothesis is that the proportion of patients who are positive for TD on both MR and pathology is not more than $13 \%$. Assuming the true proportion is equal to $26 \%$ when MRI mapping is used, then a total of 79 patients are needed to achieve $90 \%$ power. In order to conclude that the true proportion is significantly above $13 \%$, at least 16/79 patients will be required with positive results on both MR and pathology. As there is no previous data to confirm our hypothesis, we will aim to recruit 100 patients to give us a degree of safety and avoid a type 2 error. This will give us $85 \%$ power to detect an increase of $11 \%$.

The molecular pathology and genetic work is at a pilot stage only therefore a power calculation is not necessary.

\section{Analysis methods}

The total number of patients consented will be reported and any patients not having completed MRI and surgery will not be included in any further analysis and will be withdrawn from the trial. Throughout the analysis, equivocal results will be treated as equivalent to definite positive results on both pathology and MRI. For all endpoints (apart from agreement between central and local pathology review), pathology results from central review only will be used. For all MRI endpoints, only results of the central review will be used.
Continuous data will be summarised using descriptive statistics (mean, median, SD, quartiles, minimum and maximum) and categorical data will be summarised using counts and percentages. The proportion of patients with TD detected on both pathology and MRI out of total number of patients who had surgery will be given with a $95 \%$ CI. A one-sided exact binomial test of the null hypothesis that this proportion is not more than $13 \%$ will be done, with significance threshold 0.05 , as our primary endpoint. Patients with missing pathology results or no MRI central review will not be used in the primary endpoint and will be replaced in order to achieve at least 79 evaluable patients. Overall agreement between MRI and pathology will be reported as a percentage with $95 \% \mathrm{CI}$ as well as sensitivity and specificity of MRI compared with gold standard of pathology central review in diagnosing TD.

Time to local recurrence, disease free survival and overall survival will be measured from date of surgery. Time to local recurrence and disease free survival will be calculated using only patients who achieved disease free status following treatment. Overall survival will be calculated in all patients who had surgery.

Time to local recurrence will be measured until the first documented instance of local recurrence,defined as recurrence within the pelvis. Death will not be treated as an event. Patients without local recurrence will be censored at date of last known follow-up or death.

Disease free survival will be measured until the first documented instance of disease recurrence at any site or to death from any cause. Patients surviving without disease will be censored at date of last known follow-up.

Overall survival will be measured to death from any cause, surviving patients will be censored at date of last known follow-up.

Kaplan-Meier methods will be used to produce illustrative graphs, medians and estimates of 1-year, 3-year and 5-year survival (with 95\% CIs). Univariate Cox regression will be used to estimate HRs with $95 \%$ CIs associated with MRI and pathology diagnosis of TD for all three time to event endpoints. If the sample size and number of events allow, exploratory multivariate models may be used to investigate the individual contribution that TD make to prognosis when controlling for other prognostic factors such as LN status and T stage.

Results relating to interobserver agreement, objective differentiation between LN and TD (L and E scores) and whether additional pathology techniques are necessary will be descriptive only. Results relating to the use of neoadjuvant therapy and outcomes in patients with and without TD will also be descriptive only at this point but may help inform future trials.

\section{ETHICS AND DISSEMINATION}

\section{Regulatory and ethical issues}

The trial has received approval from the South CentralHampshire B Research and Ethics Committee (IRAS 217627). 
The trial will be conducted in compliance with the protocol, standard operating procedures, policies, local R\&D management guidance, Good Clinical Practice including the Research Governance Framework 2005 (second edition) and other applicable regulatory requirement(s) including but not limited to the Human Tissue Act 2004, Human Tissue (Quality and Safety for Human Application) Regulations 2007, the Medical Devices Regulations 2002, Ionising Radiation (Medical Exposures) Regulations 2000 as amended from time to time.

Data will be anonymised using unique patient identification numbers. No other patient identifiable information will be kept, other than contact details if a patient would like to be informed of the outcome of the study. All information will be encrypted and password protected, and held on the Royal Marsden computer network. Where it is necessary to move data between sites, this will be transferred between a secure fax machine or a secure nhs.net account.

\section{Patient and public involvement}

This trial and the patient information sheet (online supplemental file) were developed with direct input from members of the Surrey and South London Bowel Cancer Support Group. The proposal for the trial was discussed at one of their regular meetings and advice was sought about the perceived usefulness of the trial and acceptability to patients with very positive feedback.

\section{Dissemination}

The results of this trial will be disseminated through peerreviewed publication, even in the case of negative results. A number of national and international presentations are also anticipated. Results will also be presented to the patient support group involved in the development of the trial and as part of patient and public involvement events at the Royal Marsden Hospital.

\section{Twitter Amy C Lord @amylord1 and Gina Brown @prof_gina_brown}

Contributors ACL and GB conceived of the trial. BM, MA and SR initiated the study design and helped to implement it. IDN and MT provided pathology expertise in designing the trial and will carry out central reviewing of pathology samples. All authors contributed to refining the protocol and have read and approved the final version. All authors will be involved in analysis of results and future publications.

Funding This trial was supported by a grant from the Pelican Foundation which covered the cost of a trial manager and tissue collector. The trial also receives funding as an NIHR CRN Portfolio interventional trial.Sponsor: The Royal Marsden Hospital Foundation Trust, Clinical Research and Development, Downs road, Sutton, SM2 5PT (CCR4723)

Competing interests None declared.

Patient and public involvement Patients and/or the public were involved in the design, or conduct, or reporting, or dissemination plans of this research. Refer to the Methods section for further details.

Patient consent for publication Not required.
Provenance and peer review Not commissioned; externally peer reviewed.

Open access This is an open access article distributed in accordance with the Creative Commons Attribution Non Commercial (CC BY-NC 4.0) license, which permits others to distribute, remix, adapt, build upon this work non-commercially, and license their derivative works on different terms, provided the original work is properly cited, appropriate credit is given, any changes made indicated, and the use is non-commercial. See: http://creativecommons.org/licenses/by-nc/4.0/.

ORCID iD

Amy C Lord http://orcid.org/0000-0001-5579-6171

\section{REFERENCES}

1 Nitsche U, Maak M, Schuster T, et al. Prediction of prognosis is not improved by the seventh and latest edition of the TNM classification for colorectal cancer in a single-center collective. Ann Surg 2011;254:793-801.

2 Krook JE, Moertel CG, Gunderson LL, et al. Effective surgical adjuvant therapy for high-risk rectal carcinoma. $N$ Engl J Med 1991;324:709-15.

3 Gunderson LL, Jessup JM, Sargent DJ, et al. Revised Tn categorization for colon cancer based on national survival outcomes data. J Clin Oncol 2010;28:264-71.

4 NICE. Colorectal cancer overview - NICE Pathways, 2016. Available: https://pathways.nice.org.uk/pathways/colorectal-cancer [Accessed 15 Dec 2016].

5 Gabriel WB, Dukes C, Bussey HJR, et al. Lymphatic spread in cancer of the rectum. Br J Surg 1935;23:395-413.

6 Sobin LH, Fleming ID. Tnm classification of malignant tumors, fifth edition (1997). Cancer 1997;80.

7 Sobin LH WC. International Union against cancer TNM classification of Malig- nant tumours (ED 6. Hoboken, NJ: John Wiley \& Sons, 2002.

8 Sobin LH, Gospodarowicz MWC. International Union against cancer TNM Clas- sification of malignant tumours (ED 7. Hoboken, NJ: Wiley-Blackwell, 2009

9 Nagtegaal ID, Quirke P. Revised staging: is it really better, or do we not know? JCO 2010;28:e397-8.

10 Nagtegaal ID, Knijn N, Hugen N, et al. Tumor deposits in colorectal cancer: improving the value of modern Staging-A systematic review and meta-analysis. J Clin Oncol 2017;35:1119-27.

11 Taylor FGM, Quirke P, Heald RJ, et al. Preoperative magnetic resonance imaging assessment of circumferential resection margin predicts disease-free survival and local recurrence: 5-year follow-up results of the mercury study. J Clin Oncol 2014;32:34-43.

12 Smith NJ, Barbachano Y, Norman AR, et al. Prognostic significance of magnetic resonance imaging-detected extramural vascular invasion in rectal cancer. Br J Surg 2007;95:229-36.

13 Sohn B, Lim J-S, Kim H, et al. Mri-Detected extramural vascular invasion is an independent prognostic factor for synchronous metastasis in patients with rectal cancer. Eur Radiol 2015;25:1347-55.

14 Chand M, Evans J, Swift RI, et al. The prognostic significance of postchemoradiotherapy high-resolution MRI and histopathology detected extramural venous invasion in rectal cancer. Ann Surg 2015;261:473-9.

15 Messenger DE, Driman DK, Kirsch R. Developments in the assessment of venous invasion in colorectal cancer: implications for future practice and patient outcome. Hum Pathol 2012;43:965-73.

16 Lord AC, D'Souza N, Pucher PH, et al. Significance of extranodal tumour deposits in colorectal cancer: a systematic review and metaanalysis. Eur J Cancer 2017;82:92-102.

17 Rock JBet al. Debating deposits: an inter-observer variability study of lymph nodes and pericolonic tumor deposits in colonic adenocarcinoma. , 2014: 138, 636-42.

18 Knijn N, Mekenkamp LJM, Klomp M, et al. Kras mutation analysis: a comparison between primary tumours and matched liver metastases in 305 colorectal cancer patients. Br J Cancer 2011;104:1020-6.

19 The Royal College of pathologists. Dataset for colorectal cancer histopathology reports. $R$ Coll Pathol Guidel 2014:1-33. 